\title{
Diagnostic Testing in Acute Aortic Dissection
}

\author{
Siobhan Coyle • Tadgh Moriarty • Laura Melody • \\ Damien Ryan
}

Published online: 19 April 2014

(c) Springer Science+Business Media New York 2014

\begin{abstract}
Acute aortic dissection (AAD) is the most common catastrophe to involve the aorta, resulting in high morbidity and mortality. Delayed diagnosis can adversely affect patient outcome, therefore a high clinical index of suspicion is the first step. Absence of the classical signs such as pulse defecit and chest radiograph changes should not falsely reassure clinicians. Availability of a biomarker to expedite and improve diagnosis of AAD would greatly benefit emergency department clinicians. Some promising novel biomarkers include calponin and elastin, but their use in everyday practice is still some time away. Bedside imaging including transthoracic and transesophageal echo is being increasingly used in the unstable patient suspected of AAD, while computed tomography (CT) appears to be the most accurate rapid imaging modality for its diagnosis. Expeditious diagnosis is crucial to improve patient survival allowing for better outomes.
\end{abstract}

Keywords Acute aortic dissection - Biomarkers . Imaging $\cdot$ TEE · MRI

\section{Introduction}

Acute aortic dissection (AAD) as a clinical emergency was first described by Morgagni more than 200 years ago [1].

S. Coyle $\cdot$ T. Moriarty $\cdot$ L. Melody $\cdot$ D. Ryan $(\bowtie)$

Retrieval, Emergency and Disaster Medicine Research and Development Unit (REDSPoT), Emergency Department, University Hospital Limerick, Dooradoyle, Limerick, Ireland e-mail: damien.ryan@hse.ie

D. Ryan

Centre for Prehospital Research, Graduate Entry Medical

School, University of Limerick, Limerick, Ireland
The early detection of AAD is paramount given its significant morbidity and mortality. Recent studies have reported early mortality rates in the region of 18-25\% with little change over the past decade despite increasing technology [2]. Calculating the true incidence of AAD is difficult due to the number of aortic catastrophies that result in sudden death and go undiagnosed. A large Swedish population-based study (1982-2002) has placed the incidence over a 16-year period at 3.4 cases per 100,000 per year [3]. Early diagnosis and instigation of treatment is hampered by delayed or misdiagnosis in up to $40 \%$ of cases. This may be due to the lack of specificity and sensitivity of symptoms, signs, EKG and chest radiographs [4]. For Type A dissection, the untreated mortality is currently $1-2 \% / \mathrm{h}$ with a 30 -day mortality rate for the majority of patients approaching $100 \%$ [2]. Current evidence purports that diagnostic evaluation of patients further delays the definitive diagnosis by a number of hours, thereby increasing mortality [5].

This article aims to review the current evidence regarding the diagnosis of $\mathrm{AAD}$ in order to improve our management of this important condition.

\section{History and Clinical Diagnosis}

Although often presenting with the classical tearing chest pain radiating through to the back, AAD can present in any number of guises making it a challenging clinical diagnosis. Other symptoms and signs are often secondary to vascular compromise including pulse deficits, renal impairment and neurological signs, which can often mimic acute stroke. Less common symptoms include syncope, acute congestive heart failure and evidence of myocardial ischemia [2]. Risk factors for AAD must be taken into 
account on initial presentation and heighten clinical suspicion if present.

The most commonly associated factors are hypertension, atherosclerosis and previous cardiovascular surgery. Howard et al., in a population-based study of aortic dissection, reported $67.3 \%$ of their study population having a known history of hypertension [6••]. AAD is more frequent in the 60th decade of life with a mean age of 63 at the time of diagnosis [2]. Men are more commonly affected, accounting for $2 / 3$ of patients in the IRAD cohort (International Registry of Acute Aortic Dissection). Marfan's disease results in approximately $5 \%$ of all cases of aortic dissection, with a small number of other collagen vascular diseases (including Ehler-Danlos syndrome) causing an even smaller percentage of aortic dissections. Bicuspid aortic disease also carries a high lifetime risk of AAD with 1 in 20 patients with a bicuspid aortic valve developing AAD [7]. Cocaine has been recognized as a cause of AAD with the proposed mechanism mediated through profound catecholamine-induced elevation of the BP resulting in intimal tear [8].

Abrupt onset pain still remains the most common presenting clinical symptom. Hagan et al. conducted a case series from 1996-1998 in which patients with AAD at presentation were enrolled from 12 international centers. Of the 464 patients enrolled in this study, $84.8 \%$ of patients reported abrupt onset pain. The majority of patients complained of chest pain $(72.7 \%)$. Anterior chest pain was typical in patients with type A dissection, whereas patients with type B dissection more often experienced pain in the back and abdomen, although there was substantial overlap $(P<0.001)$ [2].

A recent study by Lovy et al. sought clinical and diagnostic criteria to identify low-risk patients as an initial step in developing a clinical decision rule in Acute Aortic Syndome (AAS). AAS included aortic dissection, rupture, intramural hematoma, and penetrating atherosclerotic ulcer. They retrospectively reviewed their institutional database for all adults initially presenting from January 1, 2006, to August 1, 2010, who underwent a CT scan for suspected AAS and who did not have a history of trauma, AAS, or aortic surgery. A total of 1,465 patients were included.

Chest pain, acute onset of pain, radiation to the back, and severe pain were all significant positive predictors of AAS. Acute chest pain had a sensitivity of $82.9 \%$ with CI 66.4-94.4\%, a specificity of $70.7 \%$, a positive predictive value (PPV) of $0.07 \%$ and a negative predictive value (NPV) of $99 \%$ [9••]. A review paper by Golledge et al. demonstrated similar results with $85 \%$ of the patients included presenting with chest or back pain [10].

Pulse deficits and the murmur of aortic regurgitation are clinical signs which are classically associated with AAD.
Pulse deficit was reported in $44 \%$ of the review patients [10]. Chua et al. conducted a retrospective chart review of 68 patients with AAD, highlighting that $63.8 \%$ did not display a pulse deficit on clinical examination. This study also showed that emergency physicians are more likely to miss the diagnosis of AAD when pulse deficit was not detected on examination (OR 35.76; $95 \%$ CI 3.70-345.34) [11•]. From IRAD aortic regurgitation and pulse deficit were noted in only $31.6 \%$ and $15.1 \%$ of patients, respectively [2]

Hypertension is a common presenting finding however inter-arm blood pressure differences are not commonly recorded. IRAD reported $49 \%$ of patients were hypertensive at presentation with hypertension at initial presentation being more common among patients with type $\mathrm{B}$ dissection (70.1 vs $35.7 \%, P<0.001$ ) [2].

Neurological deficit is widely reported with some cases presenting with acute neurological findings in the absence of pain $[12,13]$. IRAD reports overall figures of $4.7 \%$ of patients with an acute neurological deficit at presentation [2] with similar studies reporting figures of $12 \%$ [10].

In isolation, chest pain and indeed the nature of the pain may not be useful in aiding clinical diagnosis, but, in correlation, other clinical findings, patient risk factors such as known hypertension or the presence of conditions such as Marfans' syndrome and X-ray findings may aid diagnosis. Attempts have been made to formulate pathways to identify high-risk patients that require imaging. The American Heart Association have published guidelines which identify high-risk clinical features to expedite a diagnosis [14•]. This grouped risk factors (e.g. Marfan syndrome) with clinical features (tearing chest pain) and signs (pulse deficit) to risk stratify patients to immediate imaging. This guideline has since been applied to the IRAD database and is highly sensitive. However, this study did not allow for any testing of the specificity of the study. Widespread implementation of such a guideline may result in over-investigation of patients [15••].

As AAD has many mimics and up to $10 \%$ of patients may be pain free at presentation, a high clinical index of suspicion must be kept. The presence of signs such as pulse deficits, neurological symptoms and interim BP difference all raise the clinical suspicion of AAD; however, their absence does not exclude the diagnosis as evidenced by the relevant figures above.

\section{Investigations}

A high index of clinical suspicion currently remains key to the diagnosis of AAD. There is no point of care biochemical test available at present to accurately diagnose aortic dissection. Some novel biomarkers are currently 
showing promise but are unlikely to impact on improving the time to diagnosis.

\section{Current Biomarkers}

\section{Reactive Protein (CRP)}

This is an acute phase protein which rises in response to inflammatory processes. Schillinger et al. found that CRP (and white cell count) were higher in those patients presenting with chest pain and subsequently diagnosed with AAD. However, due to its poor specificity, the rise was not found to be sufficient to alter diagnostic pathways [16].

\section{D-Dimer}

The likely usefulness of this fibrin degradation product is in its "rule out" and risk stratification ability. When the dissection involves a coronary ostium and results in myocardial infarction (MI), elevated troponin and characteristic EKG changes may force the ED physician into treatment of an MI with anticoagulation therapy which would be disastrous for an AAD. The elevation of D-dimers coupled with an elevation in troponin should force the physician to rethink the diagnosis. This is because an analysis of more than 700 patients with MI showed no correlation between raised D-dimers and MI [17].

The sensitivity of this biomarker has frequently been reported as approaching $100 \%$ with a NPV of $>97 \%$ [18]. A recent meta-analysis of 349 cases provided a pooled sensitivity result of $94 \%$ [19]. False negatives were most likely in patients under 70, dissections which are shorter in length, and those with a thrombosed false lumen [20].

Despite its limitations, the use of D-dimer testing is recommended by the task force of European Society of Cardiology in the initial workup of those patients suspected of AAD [4]. A new point of care rapid latex agglutination test of whole blood providing a result within 10 min was shown to correlate well with laboratory testing [21]. This could have practical applications for usage within the ED as a screening tool when combined with an appropriate clinical presentation. Such a strategy will require research and validation before it is adopted given the likely utilization of resources.

\section{Potential New Biomarkers}

Smooth Muscle Myosin Heavy Chain (smMHC)

Research as early as 1995 by Katoh et al. suggested the use of this biomarker as a diagnostic tool for AAD [22, 23]. Levels rose rapidly within the first $24 \mathrm{~h}$ and, when a cutoff value of $2.5 \mathrm{ng} / \mathrm{l}$ was used, the specificity was $90 \%$ at $12 \mathrm{~h}$ and $85 \%$ at $24 \mathrm{~h}$. Those below the cutoff value who were subsequently diagnosed with AAD had DeBakey type B aortic dissection. At the $2.5-\mathrm{ng} / \mathrm{ml}$ cutoff, the biomarker had a specificity of $83 \%$, but at $10 \mathrm{ng} / \mathrm{ml}$ the specificity rose to $100 \%$. Interestingly, acute MI was found not to cause a rise in smMHC [24]. It must be noted that smMHC is present in uterine and intestinal smooth muscle. Theoretically, it could therefore be raised in conditions affecting these organs.

\section{Calponin}

Of the three isoforms (acidic, basic and neutral), two have shown some potential. During the first $6 \mathrm{~h}$ after AAD acidic calponin, at a value of $2.3 \mathrm{ng} / \mathrm{ml}$, demonstrated a sensitivity of $50 \%$ and specificity of $87 \%$, while the basic isoform provided sensitivity of $63 \%$ and specificity of $73 \%$ when using a cutoff value of $159 \mathrm{ng} / \mathrm{ml}$. The PPV were 0.56 and 0.44 for acidic and basic calponin, respectively, at $6 \mathrm{~h}$. The NPV of 0.84 (acidic) and 0.86 (basic) were of more clinical significance [25]. A drawback of the acidic isoform is its presence in neurological tissue, which therefore limits its accuracy for diagnosis in those with neurological signs.

\section{Elastin (sELAF)}

This biomarker measures the degradation product of elastin, one of the arterial wall's main structural components. The study used a cutoff level of 3 standard deviations above mean at all ages and resulted in a specificity of $99.8 \%$. However, this figure fell to $88.9 \%$ for those with a patent or partially thrombosed false lumen. Of significance, sELAF was negative in those with a completely thrombosed false lumen. This was one of the marker's greatest limitations. A clear benefit above calponin and smMHC was that sELAF remained raised for up to $72 \mathrm{~h}$ post-dissection [26].

Novel biomarkers are unlikely to be available to EDs in the near future, and thus are unlikely to impact significantly on early diagnosis of AAD. Of those biochemical markers readily available, D-dimer would appear to be of sufficient specificity to prompt further diagnostic evaluation and imaging to facilitate expeditious diagnosis and treatment [27].

\section{EKG}

The EKG is often the first piece of diagnostic information obtained on patients with potential AAD. In IRAD, the EKG was reported as normal in $31 \%$ of its 464 patients. Nonspecific ST and T wave changes were demonstrated in $42 \%$ with evidence of ST elevation in $5 \%$ of cases [2]. A 
retrospective study of 233 cases further confirms these findings. It demonstrated acute ST elevation in $4 \%$ of cases. ST segment depression or $\mathrm{T}$ wave inversion were seen in $47 \%$ of cases [28], while $30 \%$ of EKGs had no significant finding. The main pitfall of misdiagnosing AAD as ACS include the instigation of potentially harmful interventions such as anticoagulation, antiplatelet therapy and emergency catheter intervention. The mortality can climb to over $70 \%$ from administration of thrombolytic agents, manifested mainly by hemorrhage into the pericardial sac resulting in cardiac tamponade [29].

The underlying cause of EKG abnormalities involves hypoperfusion of the coronary arteries. A number of mechanisms have been suggested. These include a bulging false lumen which can cause occlusion of the coronary orifice, a dissection which can extend into the wall of the coronary artery, or the coronary artery itself can detach from the aortic root. In attempting to identify the vessel involved, retrospective studies have broken down the cases of ST elevation to suggest mainly right coronary artery and left main branch involvement [28].

An important aspect of EKG changes in the setting of aortic dissection is their implication for the patient's outcome. Patients with ST segment changes had a higher prevalence of pericardial effusion, cardiac tamponade, moderate/severe aortic regurgitation, and shock on admission, resulting in higher in-hospital mortality. On multivariate analysis, EKG changes were the only independent predictor of mortality. Even when the cases with ST elevation were removed from the equation, there remained an association with EKG changes and a higher in-hospital mortality [30•].

\section{Imaging}

The implications of a diagnosis of aortic dissection mandates that the imaging technique used be highly specific and sensitive. It should also provide adequate information to plan for an emergent therapeutic strategy. Planning for a therapeutic strategy depends not only on the type of dissection but also on the site of entry, the extent of dissection, the involvement of the coronary arteries, arch branches, or visceral arteries, the involvement of the aortic valve, the presence and extent of pericardial effusion, false lumen patency, and the presence of thrombus in the false lumen. Therefore, delineation of these features should be an important part of the diagnostic workup for patients with suspected aortic dissections. Imaging techniques range from the initial radiograph through to MRI.

\section{Chest X-ray}

The classic finding with aortic dissection on $\mathrm{x}$-ray is mediastinal widening. The mediastinum can be seen to bulge to the right with dissection of the ascending aorta and to the left with dissection of the thoracic aorta. Multiple other signs have been reported in cases of aortic dissection including widening of the aortic knob, aortic wall thickness, double aortic shadow and the presence of a pleural effusion, mainly on the left $[31,32]$. However, while the absence of findings on a chest $\mathrm{x}$-ray lowers the likelihood of the disease, it does not exclude its presence. In IRAD, absence of mediastinal widening was noted in $37.4 \%$ of patients presenting with type A aortic dissection. No chest x-ray abnormality was noted in $12.4 \%$ of patients [2]. Pooled data from 10 studies placed the predictive sensitivity of a widened mediastinum and abnormal aortic contour at 64 and $71 \%$, respectively. If all chest x-ray abnormalities were included, this increased the sensitivity to $90 \%$; however, this included minor chest $\mathrm{x}$-ray changes including small pleural effusions [33]. A further prospective trial showed that the specificity of a chest radiograph for significant aortic disease was $86 \%$ [34].

These studies demonstrate that the absence of chest x-ray abnormalities makes the likelihood of significant aortic disease less likely. However, it is not powerful enough to be used alone as a rule-out test. Additional studies are required in almost all patients. In unstable patients, obtaining a chest $\mathrm{x}$-ray can further delay the institution of appropriate imaging and treatment

\section{CT}

CT is well established as the most used diagnostic modality in cases of suspected AAD. IRAD reported CT as the first imaging modality used in $61 \%$ of cases [2]. Advantages of CT include its near universal availability and its speed of diagnosis. It can also delineate branch vessel involvement and visualize the entire aorta. A further plus of CT is that an alternative cause for chest pain has been reported in up to $21 \%$ of cases scanned for suspected AAD [35].

Non-helical CT scanners were more than capable of diagnosing aortic dissection in the past. However, these machines were relatively slow and any patient motion resulted in significant artefact. The sensitivity of studies using non-helical CT have been shown as close to $100 \%$ but the specificity ranges from 87 to $100 \%$. The use of helical CT has improved this reported specificity. A systematic review of 1,139 patients in 16 studies suggested that helical CT had a sensitivity of $100 \%$ and a specificity of $98 \%$ in the diagnosis of aortic dissection [36]. Multidetector CT (MDCT) has added further speed and thus improved the use of CT as a diagnostic tool. This involves using multiple detectors to obtain simultaneous images of any part of the body in a single breath-hold. MDCT allows accurate imaging of a large area in a short acquisition time with high resolution. This improves the visualization of 
vascular structures as compared with conventional CT. It also facilitates breath holding, thus minimizing artefacts on images. A study on 57 patients showed sensitivities and specificities of up to $100 \%$ with MDCT [37].

A further advancement on CT imaging has been the use of EKG gating. Movement of the heart throughout the cardiac cycle can produce motion artefacts in the image. The difficulty with interpreting cardiac motion can be helped with EKG gating. This is where data are only acquired during a specified portion of the cardiac cycle, typically during diastole. Images are created over a sequence of cardiac cycles, e.g., $\mathrm{R}$ to $\mathrm{R}$ intervals. This has been shown to be valuable for reducing ascending aortic motion artefacts that can mimic dissection without increasing imaging time [38, 39].

Triple rule-out CTs are being used more and more by various institutions allowing visualization of pulmonary arteries, aorta and coronary arteries in a single exam. This modality may safely eliminate the need for further investigation in $75 \%$ of patients in the appropriate population [40]. The major disadvantages of this protocol are the use of increased contrast with a higher radiation dose. This limits its applicability to a unique subset of patients in which AAD, pulmonary embolism and acute coronary syndrome cannot be reliably distinguished based on clinical history. Most triple rule-out CTs also do not include the abdominal aorta, thus the extent of a descending AAD may not be visualized. Continued clinical research is needed to ascertain the place of triple rule-out CTs in the investigation of chest pain and AAD.

$\mathrm{CT}$ is an effective method of diagnosing an AAD with the completion of studies analyzing its effectiveness failing to maintain pace with the speed of increasing technology.

\section{MRI}

MRA can be considered a very accurate tool for the diagnosis of AAD allowing the visualization of the aorta without the need for ionizing radiation. Both the sensitivity and specificity have been reported as $100 \%$ [36]. It is extremely accurate at identifying the site of entry, identifying thrombus and the presence of a pericardial effusion. Advantages include the assessment of functional cardiac information including left ventricular function and aortic regurgitation. There are significant limitations in the use of MRA as a first-line diagnostic tool, resulting in its use in only $1 \%$ of cases in IRAD [2]. The availability of MRI and the length of time needed for a scan limit its use in everyday practice. The monitoring of critically ill patients is also much more difficult in the MRI environment. Faster scanners are currently being explored and, as the new generations of scanners are developed, MRA may be expected to play a more prominent role [41].
Transthoracic Echo

Although the current guidelines from the AHA would point to CT as the modality of choice in ruling out AAD [4], transthoracic echo can often be overlooked as a screening tool. It is portable, inexpensive, safe and can be of particular benefit in the patient who is hemodynamically unstable. While an ascending aortic flap is diagnostic of AAD, other 'high-risk' features include aortic regurgitation, dilated aortic root (especially with a pericardial effusion), inferior hypokinesia, and a bicuspid aortic valve. As well as establishing a diagnosis of AAD, it can detect signs suggestive of an alternative diagnosis such as pericardial effusions, right heart dilatation (pulmonary embolus), and regional wall abnormalities (MI) [42].

High-risk features for type A dissection on echo can prompt ED physicians to make timely referrals to cardiothoracic surgery or arrange urgent transfer to an appropriate center. This minimizes delays in transfer of the patient within a facility, such as to and from the radiology department, which is of particular importance in the hemodynamically unstable patient, in smaller centers with no cardiothoracic unit, and in those centers with limited access to CT scanners.

For the most time-critical dissection, type A, sensitivity is $78-100 \%$. For the less urgent type B dissection, sensitivity decreases to $31-55 \%[43,44]$.

\section{Transesophageal Echo (TEE)}

The current guidelines from the European Society of Cardiology advocate the use of transesophageal echo for those hemodynamically unstable patients either prior to transfer or on arrival in the operating theatre [4]. The paradigm of AAD on TEE is an intimal flap. Reverberation artefacts have been reported to cause some misdiagnosis; however, the use of color flow imaging can help to recognize reverberation artefacts [42]. For those patients with Type A dissection, TEE can provide additional information to assist in preoperative planning. This includes coronary, head or neck vessel involvement, presence of aortic regurgitation, site of entry tear, and the proximal extent of the dissection flap. It may also detect pericardial effusion or cardiac tamponade and make an assessment of left ventricular function [45].

The sensitivity of TEE is $94-100 \%$ with a specificity of $77-100 \%$ for identifying the intimal flap. One meta-analysis concluded it had similar sensitivity and specificity for AAD detection of helical CT and MRI [36]. However, the accuracy of TEE is also dependent on the operator with its availability limited in many settings.

It must be emphasized that the absence of such factors does not rule out the presence of an aortic dissection. 
The imaging modality of choice remains CT. MRI has a higher sensitivity but its availability in the emergency situation results in $\mathrm{CT}$ becoming a more amenable diagnostic tool. TTE and TEE have been included in AHA and European society of Cardiology guidelines as a tool in the unstable patient. However, this is dependent on the operator and availability with a high level of skill involved.

\section{Conclusion}

The diagnosis of AAD is challenging for the emergency physician with potentially devastating consequences. A high index of suspicion must be maintained in order to ensure expedient and accurate diagnosis. The presence of risk factors and signs including pulse deficits or neurological symptoms propel the clinician to investigate further. There is currently no single biomarker that can be used to diagnose aortic dissection, but the judicious use of D-Dimers can aid clinicians in risk stratification and research is ongoing in this field. The future may allow novel biomarkers to be incorporated into a guideline with known risk factors and clinical features. This would significantly expedite and reduce mortality from AAD. CT remains the imaging of choice with increasing input from TTE.

\section{Compliance with Ethics Guidelines}

Conflicts of Interest All authors have declared no conflicts of interest.

Human and Animal Rights and Informed Consent This article does not contain any studies with human or animal subjects performed by any of the authors.

\section{References}

Recently published papers of particular interest have been highlighted as:

- Of importance

- Of major importance

1. Acierno LJ. The history of cardiology. New York: Parthenon; 1994.

2. Hagan PG, Nienaber CA, Isselbacher EM. The international registry of acute aortic dissection (IRAD): new insights into an old disease. JAMA. 2000;283:897-903.

3. Olsson C, et al. Thoracic aortic aneurysm and dissection increasing prevalence and improved outcomes reported in a nationwide population-based study of more than 14000 cases from 1987 to 2002. Circulation. 2006;114:2611-18.

4. Erbel R, Alfonso F, Boileau C. Diagnosis and management of aortic dissection: task force on aortic dissection, European society of cardiology. Eur Heart J. 2001;22:1642-81.
5. Raghupathy A, Nienaber CA, Harris KM. Geographic differences in clinical presentation, treatment, and outcomes in type A acute aortic dissection (from the International Registry of Acute Aortic Dissection). Am J Cardiol. 2008;102:1562-66.

6. - Howard DP, et al. Population-based study of incidence and outcome of acute aortic dissection and premorbid risk factor control: 10-year results from the Oxford Vascular Study. Circulation. 2013;127(20):2031-7. A study highlighting the significance of pre-existing hypertension in a large 10 year study.

7. Elefteriades JA, et al. Thoracic aortic aneurysm: Reading the enemy's playbook. World J Surg. 2008;32:366-74.

8. Fisher A, Holroyd BR. Cocaine-associated dissection of the thoracic aorta. J Emerg Med. 1992;10:723-7.

9. • Lovy AJ, et al. Preliminary development of a clinical decision rule for acute aortic syndromes. Am J Emerg Med. 2013;31: 1546-50. A study which attempted to identify low risk patients as an initial step in the development of a clinical decision rule for the diagnosis of aortic dissection.

10. Golledge J, et al. Acute aortic dissection. The Lancet. 2008; 372(9632):55-66.

11. - Chua M, et al. Acute aortic dissection in the ED: risk factors and predictors for missed diagnosis. Am J Emerg Med. 2012;30: 1622-6.

12. Colak N, et al. Painless aortic dissection presenting as paraplegia. Tex Heart Inst J. 2012;39(2):273-6.

13. van Zeggeren $\mathrm{L}$, et al. Painless transient paraparesis as the solitary manifestation of aortic dissection. J Vasc Surg. 2011;54(5): 1481-4.

14. - Hiratzka LF, Bakris GL, Beckman JA, et al. American college of cardiology foundation/american heart association task force on practice guidelines. Guidelines for the management of patients with thoracic aortic disease. Circulation. 2010;121:e266-e369. A Guideline which identifies high-risk features to expedite a diagnosis of aortic dissection.

15. $\bullet$ Rogers AM, et al. Sensitivity of the aortic dissection detection risk score, a novel guideline-based tool for identification of acute aortic dissection at initial presentation: results from the international registry of acute aortic dissection; IRAD Investigators. Circulation. 2011;123(20):2213-8. Highlighting the high sensitivity of the guideline produced by the AHA but also outlines limitations of lack of research into specifity of same.

16. Schillinger M, Domanovits H, Bayegan K. C-reactive protein and mortality in patients with acute aortic disease. Intens Care Med. 2002;28:740-5.

17. Weber T, Hogler S, Auer J, Berent R, Lassnig E, Kvas E, et al. D-dimer in acute aortic dissection. J Am Coll Cardiol. 2004;44:804-9.

18. Lippi G, Filippozzi L, Montagnan M, Salvango GL, Guidi GC. Diagnostic value of Ddimer measurement in patients referred to the emergency department with suspected myocardial ischaemia. J Thromb Thrombolysis. 2007;25(3):247-50.

19. Marill KA. Serum D-dimer is a sensitive test for the detection of acute aortic dissection: a pooled meta-analysis. J Emerg Med. 2008;34:367-76.

20. Hazhui H, Nishimoto M, Hoshiqa M, Negoro N, Muraoka H, Murai M, et al. Young adult patients with short dissection length and thrombosed false lumen without ulcer-like projections are liable to have false negative results of d-dimer testing for acute aortic dissection based on a study of 113 cases. Circ J. 2006;70(12):1598-601.

21. Akutsu K, Sato N, Yamamoto T. A rapid bedside D-dimer assay (cardiac D-dimer) for screening of clinically suspected acute aortic dissection. Circ J. 2005;69:397-403.

22. Katoh H, Suzuki T, Hiroi Y. Diagnosis of aortic dissection by immunoassay for circulating smooth muscle myosin. Lancet. 1995;345:191-2. 
23. Katoh H, Suzuki T, Yokomori K. A novel immunoassay of smooth muscle myosin heavy chain in serum. J Immunol Meth. 1995;185:57-63.

24. Suzuki T, Katoh H, Watanabe M. Novel biochemical diagnostic method for aortic dissection: results of a prospective study using an immunoassay of smooth muscle myosin heavy chain. Circulation. 1996;93:1244-9.

25. Suzuki T, Distante A, Zizza A. Preliminary experience with the smooth muscle troponin-like protein, calponin, as a novel biomarker for diagnosing acute aortic dissection. Eur Heart J. 2008;29:1439-45.

26. Shinohara T, Suzuki K, Okada M. Soluble elastin fragments in serum are elevated in acute aortic dissection. Arterioscler Thromb Vasc Biol. 2003;23:1839-44.

27. Ranasinghe A, Bonser R. Biomarkers in acute aortic dissection and other aortic syndromes. J Am Coll Cardiol. 2010;56(19): $1535-41$.

28. Hirata K, Wake M, Kyushima M, Takashi T, Nakazato J, Mototake $\mathrm{H}$, et al. Electrocardiographic changes in patients with type A acute aortic dissection incidence, patterns and underlying mechanisms in 159 cases. J Cardiol. 2010;56(2):147-53.

29. Kamp TJ, Goldschmidt-Clermont PJ, Brinker JA, Resar JR. Myocardial infarction, aortic dissection, and thrombolytic therapy. Am Heart J. 1994;128:1234-7.

30. - Kimura K, et al. Frequency and implication of ST-T abnormalities on hospital admission electrocardiograms in patients with type A acute aortic dissection. Am J Cardiol. 2013;112(3): 424-9. Identifies the relationship between EKG changes and a higher in hospital mortality.

31. Earnest F, Muhm JR, Sheedy PF. Roentgenographic findings in thoracic aortic dissection. Mayo Clin Proc. 1979;54:43-50.

32. Jagannath AS, Sos TA, Lockhart SH, et al. Aortic dissection: a statistical analysis of the usefulness of plain chest radiographic findings. AJR Am J Roentgenol. 1986;147:1123-6.

33. Klompas M, et al. Does this patient have an acute thoracic aortic dissection? JAMA. 2002;287(17):2262-72.

34. Von Kodolitsch Y, Nienaber CA, Dieckmann C, et al. Chest radiography for the diagnosis of acute aortic syndrome. Am $\mathrm{J}$ Med. 2004;116:73-7.
35. Thoongsuwan N, Stern EJ. Chest CT scanning for clinical suspected thoracic aortic dissection: beware the alternate diagnosis. Emerg Radiol. 2002;9(5):257-61.

36. Shiga T, Wajima Z, Apfel CC, et al. Diagnostic accuracy of transesophageal echocardiography, helical computed tomography, and magnetic resonance imaging for suspected thoracic aortic dissection: systematic review and meta-analysis. Arch Intern Med. 2006;166:1350-6.

37. Yoshida S, Akiba H, Tamakawa M, et al. Thoracic involvement of type A aortic dissection and intramural hematoma: diagnostic accuracy-comparison of emergency helical CT and surgical findings. Radiology. 2003;228(2):430-5.

38. Roos JE, Willmann JK, Weishaupt D, Lachat M, Marincek B, Hilfiker PR. Thoracic aorta: motion artifact reduction with retrospective and prospective electrocardiography-assisted multidetector row CT. Radiology. 2002;222(1):271-7.

39. Schertler T, Glucker T, Wildermuth S, Jungius KP, Marincek B, Boehm T. Comparison of retrospectively ECG-gated and nongated MDCT of the chest in an emergency setting regarding workflow, image quality, and diagnostic certainty. Emerg Radiol. 2005;12(1-2):19-29.

40. Halpern EJ. Triple-rule-out CT angiography for evaluation of acute chest pain and possible acute coronary syndrome. Radiology. 2009;252(2):332-45.

41. Pereles FS, McCarthy RM, Baskaran V, et al. Thoracic aortic dissection and aneurysm: evaluation with nonenhanced true FISP MR angiography in less than 4 minutes. Radiology. 2002;223(1):270-4.

42. Meredith E, Masani N. Echocardiography in the emergency assessment of acute aortic syndromes. Eur J Echocardiogr. 2009; 10:131-9.

43. Appelbaum A, Karp R, Kiklin J. Ascending vs descending aortic dissections. Ann Surg. 1976;183:296-300.

44. Daily PO, Trueblood H, Stinson EB, Wuerflein R, Shumway N. Management of acute aortic dissections. Ann Thorac Surg. 1970; 10:237-47.

45. Evangelista A, Gonzalez-Alujas M, Garcia del Castillo H, Anivarro I, Angel J, Salas A, et al. Transoesophageal echocardiography in the diagnosis of aortic dissection. Revista Espanola de Cardiologia. 1993;46:805-9. 